\title{
Nutritional status and intestinal iron absorption in children with chronic hepatic disease with and without cholestasis
}

\author{
Regina Helena Guedes da Motta Mattar, ${ }^{1}$ Ramiro Anthero de Azevedo, ${ }^{2}$ \\ Patricia Graça Leite Speridião, ${ }^{3}$ Ulysses Fagundes Neto, ${ }^{4}$ Mauro Batista de Morais ${ }^{5}$
}

\begin{abstract}
Objective: to evaluate food intake, occurrence of energy-protein malnutrition and anemia, and intestinal iron absorption in children with chronic liver disease.

Methods: The study included 25 children with chronic liver disease, 14 with cholestasis and 11 without cholestasis. The age varied between 6.5 months and 12.1 years. Intestinal iron absorption was evaluated by the increment of serum iron one hour after the ingestion of $1 \mathrm{mg} / \mathrm{kg}$ of elemental iron and by the response to oral iron therapy. Iron intestinal absorption was compared to a group with iron deficiency anemia (without liver disease).

Results: The mean intake of energy and protein in the cholestatic group was higher than in patients without cholestasis. The nutritional deficit was more severe in cholestatic patients, especially with regard to height-for-age and weight-for-age indices. Anemia was found in both cholestatic group $(11 / 14 ; 78.6 \%)$ and noncholestatic group $(7 / 11$; $63.6 \%)$. The cholestatic group presented lower $(p<0.05)$ intestinal iron absorption $(90.6 \pm 42.1 \mu \mathrm{g} / \mathrm{dl})$ than the iron deficiency anemia group $(159.6 \pm 69.9 \mu \mathrm{g} / \mathrm{dl})$. However, cholestatic patients responded to oral iron therapy. The noncholestatic group showed intestinal iron absorption similar to the iron deficiency anemia group.

Conclusion: The cholestatic group showed more severe nutritional deficits. Despite the evidence of lower iron intestinal absorption, cholestatic patients responded to oral iron therapy, probably, due to the coexistence of iron deficiency.

J Pediatr (Rio J). 2005;81(4):317-24: Nutritional status, anemia, intestinal absorption, iron, inflammation, liver diseases.
\end{abstract}

\section{Introduction}

Nutritional deficiencies are common among children with chronic liver disease, especially when the process is cholestatic and has onset before 6 months. ${ }^{1}$ Severe proteinenergy malnutrition affects approximately $60 \%$ of children with chronic liver disease, ${ }^{2-5}$ being secondary to the

1. MSc. Physician, Universidade Federal de São Paulo - Escola Paulista de Medicina (UNIFESP/EPM), São Paulo, SP, Brazil.

2. PhD. Associate professor, UNIFESP/EPM, São Paulo, SP, Brazil.

3. PhD. Visiting professor, UNIFESP/EPM, São Paulo, SP, Brazil.

4. Professor, UNIFESP/EPM, São Paulo, SP, Brazil.

5. Full professor, UNIFESP/EPM, São Paulo, SP, Brazil.

Manuscript received Dec 23 2004, accepted for publication Mar 302005.

Suggested citation: Mattar RH, de Azevedo RA, Speridião PG, Fagundes Neto $U$, de Morais MB. Nutritional status and intestinal iron absorption in children with chronic hepatic disease with and without cholestasis. J Pediatr (Rio J). 2005;81:317-24. interaction of multiple factors, including reduced nutritional intake, intestinal malabsorption and increased nutrient requirements due to increased energy expenditure. 1,6,7 Despite the knowledge that an adequate nutritional status contributes towards successful liver transplantation and lower rates of postoperative complications, ${ }^{5,8-12}$ studies performed during transplant pre-op show that a large proportion of patients with chronic liver disease exhibit inadequate nutrient intake and protein-energy malnutrition. 6,12

Another common manifestation of chronic liver disease is anemia. The greater part of what is known about anemia in chronic liver disease comes from studies of patients with alcoholic cirrhosis. The principal factors involved in its genesis are: increased plasma volume, hemorrhages via esophageal vessels, hemolysis, deficiencies of elements such as iron, vitamin B-12 and folic acid and abnormalities linked to chronic 
inflammation. 13 Many of these factors are common to anemia of chronic inflammation, for which reason it has been proposed that anemia of chronic liver disease be included in this syndrome group. In addition to the reduced life expectancy of blood cells and the reduction in utilization of iron sequestered within the reticuloendothelial system, with anemia of chronic inflammation, there can also be intestinal iron malabsorption. Studies performed as our service employed the serum iron increase test and oral iron therapy response test as indicators of intestinal iron absorption. ${ }^{14,15}$ It should be pointed out that, as a result of the elevated prevalence of iron deficiency anemia in our country, iron deprivation should be considered as a possible aggravating factor of anemia in children with chronic liver disease. It should also be remembered that there is increased intestinal iron absorption with iron deficiency anemia and that this can be detected by the serum iron increase test. 14,15

Therefore, the objective of this study of children with chronic liver disease was to evaluate: 1 ) nutritional intake nutritional intake; 2) the presence of protein-energy malnutrition; 3 ) the presence of anemia and 4) intestinal iron intake.

\section{Patients and methods Patients}

This was a cross-sectional study, onto which were enrolled all patients seen at the Hepatology Clinic of the Universidade Federal de São Paulo - Escola Paulista de Medicina (UNIFESP/EPM) Pediatric Gastroenterology Department fulfilling the following inclusion criteria: 1) suffering from chronic liver disease as characterized by the following anatomical and pathological findings in liver biopsy: fibrosis and nodularity, altered hepatic parenchyma structure and hepatic cell abnormalities, such as regenerative hyperplasia, pleomorphism and dysplasia 16 and 2) no sign of liver decompensation (ascites, edema and hemorrhage) during the 30 days prior to the study. The following were not enrolled: 1) auto-immune liver disease patients of carriers of hepatitis B or C infections; 2) patients who had been given oral iron therapy during the previous 30 days; 3 ) patients with hemoglobin levels below $8 \mathrm{~g} / \mathrm{dl}$; 4 ) patients who had undergone surgery for biliary atresia less than 6 months previously. Patients were excluded from the study if they presented infection during the iron therapy test with ferrous sulphate or if they received less than $80 \%$ of the prescribed iron dose.

In accordance with these procedures, 25 patients were studied, nine of whom were male and 16 female. Age varied from 6.5 months to 12.1 years. The median age (25th and 75th percentiles in parentheses) of patients with cholestasis was 18 months (14.1-37) and for the group with no cholestasis this figure was 22.1 months (15.6-110.6), with the Mann-Whitney test revealing no difference between the groups $(p=0.366)$. Depending on bilirubin, patients were classified as wither cholestatic or not. Cholestasis was defined as total bilirubin above
$2 \mathrm{mg} / \mathrm{dl}$ or direct bilirubin accounting for more than $20 \%$ of total bilirubin. ${ }^{17}$ According to these criteria, 14 patients were classified as cholestatic and 11 as not suffering from cholestasis. Seventeen of these patients were diagnosed as suffering from biliary atresia after at least 6 months of portoenterostomy, three presented progressive familial intrahepatic cholestasis (Byler syndrome), one had an alpha-1-antitrypsin deficiency, one chronic hepatopathy due to histiocytosis $X$ and three liver diseases with no definitive etiologic diagnosis.

The study was approved by the UNIFESP/EPM Ethics Commission. Informed consent in writing was obtained from the patients' parents or guardians.

\section{Methods \\ Dietary assessment}

At the first consultation the children's mothers were instructed on how to complete a 72-hour dietary recall, 18,19 which they took home to complete. The software program Sistema de Apoio à Decisão em Nutrição, version 2.5 was used to calculate energy, protein and iron intake from the information provided by the dietary recall. 20 This program employs the reference standards for age given in the Recommended Dietary Allowances, RDA (National Research Council - NRC, 1989). ${ }^{21}$

\section{Anthropometry}

Weight and stature were measured according to recommendations made by Jelliffe. ${ }^{22}$ Patients were measured unclothed. Weighing was performed using one of two scales by Filizola ${ }^{\circledR}$, either for infants or larger children. For children younger than 24 months, stature was measured in decubitus dorsal with an anthropometric ruler with a movable cursor. Children older than 24 months were measured using a vertical stadiometer. 22

Triceps skin folds (SKF) were measured in millimeters with a Lange Skinfold Caliper at the posterior superior region of the relaxed, non-dominant arm at the midpoint of an imaginary line passing from the acromion to the olecranon. ${ }^{23}$ Measurements were repeated three times with the final result being the mean of the three results to an accuracy of $0.1 \mathrm{~mm}$.

Mean arm circumference was measured with a nonstretch measuring tape, encircling the mid portion of the non-dominant arm, at the midpoint of an imaginary line joining the acromion to the olecranon with the arm relaxed. ${ }^{23}$ Measurements were taken to an accuracy of $0.1 \mathrm{~mm}$.

The formula proposed by Frisancho ${ }^{23}$ was used to calculate muscular arm circumference (MAC) based on the values obtained for TSF and upper arm circumference: $(\mathrm{MAC})(\mathrm{cm})=\operatorname{UAC}(\mathrm{cm})-[3.1416 \times \operatorname{TSF}(\mathrm{mm})]$. The muscular arm area (MAA) was calculated using the formula: MAA $(\mathrm{mm})^{2}=[(\mathrm{MAC}-3.1416) \times \mathrm{TSF}]^{2} / 4 \times 3.1416 .23$

Epi-Info version $6.0^{24}$ software was used to calculate $z$ scores for weight-age, weight-stature and stature-age. 
Values below -2 standard deviations were considered indicative of an anthropometric deficit. Additionally, z-scores were calculated for TSF, upper arm circumference and arm muscular area using reference values from Frisancho ${ }^{25}$ for children more than 1 year old and values from Sann et al. ${ }^{26}$ for children less than one.

\section{Laboratory tests}

Blood counts were performed by an automated electronic counter (Cell-Dyn 3500 by Abbott) at the Hospital São Paulo's central laboratory. Anemia was defined as hemoglobin below $11 \mathrm{~g} / \mathrm{dl}$ before 4 years of age, below $11.5 \mathrm{~g} / \mathrm{dl}$ between 5 and 7 years and $12 \mathrm{~g} / \mathrm{dl}$ from 7 to 12 years. ${ }^{27}$ Serum iron levels were tested by a colorimetric method (PAK IRON ${ }^{\circledR}$ by Bayer), transferrin by the Beckman ${ }^{\circledR}$ immunochemical method and ferritin assay by the immunoenzymometric method (AIA-PACK $\mathrm{FER}^{\circledR}$ ) at the Psychobiology Laboratory at UNIFESP/EPM. Transferrin saturation was calculated based on values for serum iron and transferrin. Liver function tests were performed by conventional methods at the Hospital São Paulo's central laboratory.

\section{Intestinal iron uptake}

Serum iron absorption tests were performed after eight hours' fasting. ${ }^{14}$ A blood sample was taken to measure base serum iron levels. Immediately after the fasting sample had been taken, $1 \mathrm{mg}$ of elemental iron was administered orally per kilo of patient weight in the form of ferrous sulphate. Two hours after the base level fasting blood sample had been collected, a second sample was taken and the serum iron level measured once more. Intestinal iron intake was based on the increase in serum iron expressed in $\mu \mathrm{g} / \mathrm{dl}$ (serum iron 2 hours after ferrous sulphate administration minus fasting serum iron level). ${ }^{14}$

After the baseline tests had been performed, the treatment test was initiated with ferrous sulphate (Fer-insol ${ }^{\circledR}$, Mead-Johnson) at a daily dose of $5 \mathrm{mg} / \mathrm{kg} /$ day of elemental iron, divided into two equal parts and given via the oral route between meals. The medication was provided to patients and their consumption was gauged according to the volume consumed after 15 and 30 days' iron therapy. On the thirtieth day of oral iron therapy a blood count was performed to calculate hemoglobin increase.

For comparison of iron absorption tests (based on increase in serum iron 2 hours after ferrous sulphate ingestion) and of ferrous sulphate therapy tests, values were used that had been observed with 27 children with iron deficiency anemia and who had undergone the same diagnostic procedures. These children had been referred from the walk-in center with suspected iron deficiency anemia for diagnostic confirmation, intestinal iron uptake and oral iron therapy assessments as part of their clinical investigations. Their ages varied from 10 to 78 months, and 14 of them were male. None of these patients exhibited or had previously exhibited any clinical evidence of liver disease.

\section{Statistics}

Results were analyzed using both parametric and nonparametric statistical tests depending on the distribution of variables. Calculations were run on SigmaStat version 2.0 software. For all tests the null hypothesis rejection point was set at $5 \%$. The tests used for each analysis are described together with their results.

\section{Results}

Table 1 lists the results of liver function tests for patients with and without cholestasis. Patients with cholestasis exhibited higher values, with statistical significance, for total and direct bilirubin, for aspartate aminotransferase and for triglycerides, while their albumin was lower than for the group without cholestasis. Statistical analysis did not reveal any difference in values for alanine aminotransferase, alkaline phosphatase, gamma-glutamyl transpeptidase, prothrombin activity or total proteins.

Table 2 lists nutrient intake according to the 72-hour dietary recall. Energy and protein intake was lower among patients without cholestasis. No statistically significant difference in iron intake was detected, although it should be pointed out that the median consumption was 57 and $70 \%$, for patients with and without cholestasis, respectively.

Table 3 lists the anthropometric results. According to the z-scores, nutritional deficit was more severe, with statistical significance, among patients with cholestasis, with the exception of the arm muscle area z-score. The number of children with a stature deficit for their ages (z-score $<-2$ standard deviations) was greater (Fisher's exact test; $\mathrm{p}=0.042$ ) among patients with cholestasis $(9 / 14 ; 64.3 \%)$ than among patients without cholestasis $(2 / 11,18.2 \%)$. The same relationship was observed for weight for age deficits ( $p=0.033)$, present in $8 / 14(57.2 \%)$ of the cholestatic patients and in $1 / 11(9.1 \%)$ of those without cholestasis. No statistically significant difference in weight for stature deficit was observed, with $3 / 14$ (21.4\%) and $0 / 11(0.0 \%)$ suffering this deficiency among patients with and without cholestasis, respectively.

Table 4 lists indicators for anemia and body iron content. It was found that hemoglobin and hematocrit levels were lower among patients with cholestasis than among those patients without cholestasis. The mean values for mean corpuscular volume and serum iron were similar for the two groups. The level of serum ferritin was higher among children with cholestasis, while transferrin was lower.

Based on hemoglobin levels, anemia was identified in 11 (78.6\%) of the 14 cholestatic children and in seven (63.6\%) of the 11 children without cholestasis (Fisher's exact test, $p=0.656)$.

Table 5 contains the results of the iron absorption test, initial hemoglobin, hemoglobin on the thirtieth day of oral iron therapy and the response to oral iron therapy among the children with chronic liver disease and anemia. For purposes of comparison, results were used from iron absorption tests and oral iron therapy response tests of 
Table 1 - Results of liver function tests (median and percentiles 25 and 75 in parenthesis) in patients with chronic liver disease and with and without cholestasis

\begin{tabular}{lccc}
\hline & \multicolumn{2}{c}{ Cholestasis } & \multirow{2}{*}{ * } \\
& Yes $(\mathbf{n}=\mathbf{1 4})$ & No $(\mathbf{n}=\mathbf{1 1})$ & \\
\hline Aspartate aminotransferase (U/l) & $215(143-244)$ & $85(71-116)$ & 0.003 \\
Alanine aminotransferase (U/l) & $135(75-187)$ & $67(55-107)$ & 0.119 \\
Alkaline phosphatase (U/l) & $1,708(938-2,020)$ & $730(664-1,894)$ & 0.218 \\
Gamma-glutamyl transpeptidase (U/l) & $216(104-351)$ & $228(141-265)$ & 0.891 \\
Total bilirubin (mg/dl) & $9.6(5-13.1)$ & $1(0.7-1.5)$ & $<0.001$ \\
Direct bilirubin (mg/dl) & $6.9(4-11.6)$ & $0.7(0.4-0.8)$ & $<0.001$ \\
Cholesterol $(\mathrm{mg} / \mathrm{dl})$ & $203(140-265)$ & $184(160-225)$ & 0.848 \\
Triglycerides $(\mathrm{mg} / \mathrm{dl})$ & $214(98-239)$ & $135(62-165)$ & 0.035 \\
Prothrombin activity $(\%)$ & $77(39-98)$ & $98(72-100)$ & 0.147 \\
Total proteins $(\mathrm{g} / \mathrm{dl})$ & $7.0(6.3-7.3)$ & $6.7(6.4-7.8)$ & 0.584 \\
Albumine $(\mathrm{g} / \mathrm{dl})$ & $3.3(3-3.7)$ & $3.9(3.7-4.3)$ & 0.005 \\
\hline
\end{tabular}

* Mann-Whitney's test.

Table 2 - Nutrient intake (median and percentiles 25 and 75 in parenthesis) according to patients with chronic liver disease with and without cholestasis

\begin{tabular}{lccc}
\hline & \multicolumn{2}{c}{ Cholestasis } & \multirow{2}{*}{ p * } \\
& Yes $(\mathbf{n}=\mathbf{1 4})$ & No $(\mathbf{n}=\mathbf{1 1})$ & \\
\hline Energy intake (kcal/kg/dia) & $121(95.8-250.3)$ & $64.4(58.8-81.4)$ & 0.011 \\
Energy adequated to the RDA (\%) & $117.4(93.8-237.4)$ & $71.9(56-87.9)$ & 0.013 \\
Protein intake (g/kg/dia) & $4.6(3.4-8.2)$ & $3.4(2.2-3.9)$ & 0.033 \\
Iron intake adequated to the RDA (\%) & $57.0(28.5-88.0)$ & $70.0(55.7-82.0)$ & 0.442 \\
\hline
\end{tabular}

* Mann-Whitney's test.

27 children with iron deficiency anemia and free of chronic liver disease who had had their intestinal iron uptake assessed by the same methods. Patients with liver disease who did not exhibit anemia were not included in this analysis, since intestinal iron uptake in individuals with no iron deficiency is not elevated and, furthermore, hemoglobin levels would not be expected to increase as a result of oral iron therapy. The iron absorption test showed that the intestinal iron uptake of children with chronic liver disease with cholestasis was inferior to the iron absorption of the 27 children suffering from iron deficiency anemia and free from liver disease. It should be pointed out that, before oral iron therapy, the mean hemoglobin level of patients with chronic liver disease and cholestasis was similar to that for the group with iron deficiency anemia and that levels for both groups were below the mean of the patients with chronic liver disease, but no cholestasis. After 30 days of oral iron therapy the same mean value was observed for the groups with liver disease without cholestasis and iron deficiency anemia. With respect of the response to oral iron therapy, it was observed that patients with liver disease without cholestasis presented a lower mean hemoglobin increment than the group with anemia and that this difference was statistically significant. The paired t test found a statistically significant hemoglobin increase on the thirtieth day compared with baseline hemoglobin for all three study groups.

Table 6 lists body iron content indicators for patients with liver disease with or without cholestasis and for children with iron deficiency anemia, but no liver disease. Although the Kruskal-Wallis test indicated differences in serum iron levels, the multiple comparisons test did not confirm this difference. The median for transferrin among patients with cholestatic liver disease was lower than the medians for patients with liver disease without cholestasis 
Table 3 - Z-scores (median and percentiles 25 and 75 in parenthesis) of the anthropometric resulsts of patients with chronic liver disease with and without cholsetasis

\begin{tabular}{lccc}
\hline & \multicolumn{2}{c}{ Cholestasis } & p * \\
& Yes $(\mathbf{n}=\mathbf{1 4})$ & No $(\mathbf{n}=\mathbf{1 1})$ & \\
\hline Weight-age & $-2.50(-3.68 ;-1.28)$ & $-0.65(-1.38 ;+0.12)$ & 0.007 \\
Weight-height & $-0.85(-1.96 ; 0.00)$ & $-0.04(-0.33 ;+0.55)$ & 0.035 \\
Height-age & $-3.08(-4.00 ;-1.27)$ & $-0.69(-1.40 ;-0.28)$ & 0.009 \\
Triceps skin fold & $-1.90(-2.29 ;-1.42)$ & $-0.43(-0.96 ;-0.21)$ & 0.001 \\
Arm circumference & $-2.45(-4.20 ;-1.69)$ & $-0.54(-1.28 ;+1.00)$ & 0.010 \\
Muscular arm area & $-1.19(-2.51 ; 0.00)$ & $-0.93(-1.21 ;-0.23)$ & 0.286 \\
\hline
\end{tabular}

* Mann-Whitney's test.

Table 4 - Indicators for anemia and body iron content of patients with chronic liver disease with and without cholsetasis

\begin{tabular}{|c|c|c|c|}
\hline & \multicolumn{2}{|c|}{ Cholestasis } & \multirow[t]{2}{*}{$\mathbf{p}$} \\
\hline & Yes $(n=14)$ & No $(n=11)$ & \\
\hline Hemoglobin $(\mathrm{g} / \mathrm{dl}) *$ & $10 \pm 1.2$ & $11.2 \pm 1$ & 0.011 \\
\hline Hematocrit $(\%) *$ & $30.7 \pm 3.3$ & $33.8 \pm 2.6$ & 0.018 \\
\hline Mean corpuscular volume $\left(\mu^{3}\right) *$ & $83.1 \pm 12$ & $82.1 \pm 5.0$ & 0.796 \\
\hline Serum iron $(\mu \mathrm{g} / \mathrm{dl})^{+}$ & $55(38-74)$ & $54(47-64)$ & 0.784 \\
\hline $\operatorname{Transferrin}_{(\mathrm{mg} / \mathrm{dl})^{+}}{ }^{+}$ & $258(220-294)$ & $358(297-371)$ & 0.003 \\
\hline Transferrin saturation $(\%)^{+}$ & $23.1(16.4-29)$ & $14.5(13-22.8)$ & 0.059 \\
\hline Ferritin $(\mathrm{ng} / \mathrm{ml})^{\dagger}$ & $61(39-137.8)$ & $19(18.2-46.9)$ & 0.011 \\
\hline
\end{tabular}

* Mean \pm standard deviation, Student's t test.

$\dagger$ Median and percentiles 25 and 75 in parenthesis, Mann-Whitney's test.

and with iron deficiency anemia. The median for patients with cholestatic liver disease was higher than that of the patients with iron deficiency anemia.

\section{Discussion}

Our study, based on anthropometry findings, has shown that our patients with cholestatic chronic liver disease exhibited more severe nutritional deficit than did those with chronic liver disease, but no cholestasis (Table 3). The most extreme deficit among the cholestatic patients was stature for age (median $z$-score $=-3.08$; with $9 / 15,64.3 \%$, below -2 standard deviations). In this same subset, a significant deficit in weight for age was also observed, while the deficit in weight for stature was proportionally less severe (median z-score $=-0.85$, with $3 / 14,21.4 \%$, below -2 standard deviations). This nutritional profile is, along general lines, similar to that observed in other studies in the literature that assessed the nutritional status of children with chronic hepatopathy. $6,12,28$ Theoretically, water retention and visceromegaly may, at least in part, lead to an underestimation of weight deficit. In our study patients with clinical signs of edema or ascites were not included in order to reduce the influence of this factor on the nutritional assessment. The laboratory investigation revealed that the median albumin $(3.3 \mathrm{~g} / \mathrm{dl})$ of patients with cholestasis was lower than for the patients without cholestasis (Table 1), suggesting that patients with cholestasis could have been suffering from a certain degree of subclinical water retention. The median transferrin level, which was lower in the group with cholestasis (Table 4), is probably related to other factors, in addition to protein-energy malnutrition, such as the degree of liver failure compromising protein synthesis, the body iron content and the inflammatory process of the hepatopathy itself. Observe, in Table 2, that the ingestion of nutrients by patients with chronic liver disease is greater among those with cholestasis and that, even so, they exhibit more severe malnutrition. This result could be explained by these patients' being more compliant with their dietary guidance. Nevertheless, the median energy intake may yet 
Table 5 - Iron absorption test assessed with the test of serum iron increase and response to oral iron therapy

\begin{tabular}{|c|c|c|c|c|}
\hline & \multicolumn{2}{|c|}{ Cholestasis } & \multirow{2}{*}{$\begin{array}{c}\text { Iron deficiency } \\
\text { anemia } \\
(n=27)\end{array}$} & \multirow{2}{*}{$\begin{array}{l}\text { Variance } \\
\text { analysis }\end{array}$} \\
\hline & $\begin{array}{c}\text { Yes } \\
(n=11)\end{array}$ & $\begin{array}{c}\text { No } \\
(n=7)\end{array}$ & & \\
\hline Iron absorption test $(\mu \mathrm{g} / \mathrm{dl})$ & $90.6 \pm 42.1$ & $158.0 \pm 89.5$ & $159.6 \pm 69.9$ & 0.022 \\
\hline Initial hemoglobin ( $\mathrm{g} / \mathrm{dl})$ & $9.6 \pm 0.9$ & $10.9 \pm 0.7$ & $9.7 \pm 1.1$ & 0.030 \\
\hline Final hemoglobin $(\mathrm{g} / \mathrm{dl})$ & $10.8 \pm 1.4$ & $11.6 \pm 1.2$ & $11.6 \pm 0.9$ & 0.141 \\
\hline Response to oral iron therapy $(\mathrm{g} / \mathrm{dl})$ & $1.3 \pm 1.4$ & $0.7 \pm 0.8$ & $1.9 \pm 0.9$ & 0.028 \\
\hline
\end{tabular}

Tukey's multiple comparison test:

Iron absorption test

- iron deficiency anemia versus liver disease with cholestasis: $p<0.05$

- iron deficiency anemia versus liver disease without cholestasis: $p>0.05$

- liver disease with cholestasis versus liver disease without cholestasis: $p>0.05$

Initial hemoglobine

- iron deficiency anemia versus liver disease with cholestasis: $p>0.05$

- iron deficiency anemia versus liver disease without cholestasis: $p<0.05$

- liver disease with cholestasis versus liver disease without cholestasis: $p<0.05$

Response to oral iron therapy

- iron deficiency anemia versus liver disease with cholestasis: $p>0.05$

- iron deficiency anemia versus liver disease without cholestasis: $p<0.05$

- liver disease with cholestasis versus liver disease without cholestasis: $p<0.05$

remain below their true requirements, which would be a possible explanation of the persistent malnutrition. IT should be pointed out that the present study is not capable of evaluating the efficacy of nutritional intervention among patients with chronic liver disease. There is little published literature on the impact of nutritional intervention on chronic liver disease. However, studies 29,30 do show evident nutritional recovery after a successful liver transplantation. These comments indicate the need for improved knowledge about the alterations to the growth and nutrition of patients with chronic liver disease so that their nutritional therapy can be refined. On the other hand, current nutritional therapy unquestionably represents an advance on the past and, probably, has reduced the nutritional deficit of these patients, for which reason it is one of the most important points in the treatment of children with chronic liver disease.

Triceps skin fold and mean arm circumference deficits were significantly greater among patients with cholestasis than those observed in patients without cholestasis (Table 3 ). This result indicates an accentuated reduction in fat reserves, as has been described previously 12,28 among liver disease patients, and a tendency to preserve protein reserves in chronic liver disease malnutrition. 6

The iron absorption test, based on serum iron increase showed that the mean absorption of the group with iron deficiency anemia was greater than in the group with cholestatic liver disease (Table 5). In turn, the initial mean hemoglobin of the group with iron deficiency anemia and the patients with cholestatic liver disease were very similar, but lower $(p<0.05)$ than the mean for the group with liver disease, but no cholestasis (Table 5). All three groups exhibited a response to the iron therapy test according to the paired t test comparing initial hemoglobin levels with the thirtieth day of oral iron therapy. The mean increase $(0.7 \pm 0.8 \mathrm{~g} / \mathrm{dl})$ in the group with liver disease, but without cholestasis was less $(p<0.05)$ than for the group with iron deficiency anemia $(1.9 \pm 0.9 \mathrm{~g} / \mathrm{dl})$. Hemoglobin values on the thirtieth day of oral iron therapy resulted in the same mean for the groups with iron deficiency anemia and noncholestatic liver disease, which mean was not statistically different from the group with liver disease with cholestasis. Nevertheless, the mean hemoglobin in the group with cholestasis was $0.8 \mathrm{~g} / \mathrm{dl}$ lower than for the other two groups. Interpreting these results, taking each group as a whole and not the individual values, allows us to speculate that liver disease patients without cholestasis present anemia with iron deficiency as the predominant etiology, considering their similar iron absorption to the patients with iron deficiency anemia, the response to iron therapy, which is lower the higher the baseline hemoglobin value is ${ }^{15}$ and the hemoglobin level on the thirtieth day of iron therapy. In turn, everything indicates that the group with cholestatic liver disease also has an inflammatory component to the etiology of their anemia. This is evidenced by the higher median ferritin level, lower intestinal iron uptake, according 
Table 6 - Body iron content indicators for patients with anemia and liver disease with or without cholestasis and for children with iron deficiency anemia, but no liver disease

\begin{tabular}{|c|c|c|c|c|}
\hline & \multicolumn{2}{|c|}{ Cholestasis } & \multirow{2}{*}{$\begin{array}{c}\text { Iron deficiency } \\
\text { anemia } \\
(n=27)\end{array}$} & \multirow[b]{2}{*}{$\mathbf{p}$} \\
\hline & $\begin{array}{c}\text { Yes } \\
(n=11)\end{array}$ & $\begin{array}{c}\text { No } \\
(n=7)\end{array}$ & & \\
\hline Serum iron $(\mu \mathrm{g} / \mathrm{dL})$ & $48(39-74)$ & $57(50-63)$ & $27(13-65)$ & 0.017 \\
\hline Transferrin (mg/dL) & $233(212-294)$ & $358(297-394)$ & $415(352-465)$ & $<0.001$ \\
\hline Ferritin $(\mathrm{ng} / \mathrm{ml})$ & $64.3(45.4-123.5)$ & $18.6(14.8-43.8)$ & $2.1(0.4-16.0)$ & $<0.001$ \\
\hline Transferrin saturation (\%) & $21.8(16.424 .3)$ & $14.5(13.8-22.8))$ & $5.6(3.0-16.2$ & 0.002 \\
\hline
\end{tabular}

Dunn's multiple comparison test:

Serum iron

- iron deficiency anemia versus liver disease with cholestasis: $p>0.05$

- iron deficiency anemia versus liver disease without cholestasis: $p<0.05$

- liver disease with cholestasis versus liver disease without cholestasis: $p<0.05$

Transferrin

- iron deficiency anemia versus liver disease with cholestasis: $p>0.05$

- iron deficiency anemia versus liver disease without cholestasis: $p<0.05$

- liver disease with cholestasis versus liver disease without cholestasis: $p<0.05$

Ferritin

- iron deficiency anemia versus liver disease with cholestasis: $p>0.05$

- iron deficiency anemia versus liver disease without cholestasis: $p<0.05$

- liver disease with cholestasis versus liver disease without cholestasis: $p<0.05$

Ferritin saturation

- iron deficiency anemia versus liver disease with cholestasis: $p>0.05$

- iron deficiency anemia versus liver disease without cholestasis: $p<0.05$

- hepatic disease with cholestasis versus hepatic disease without cholestasis: $p>0.05$

to serum iron increase, and the lower mean hemoglobin on the thirtieth day of oral iron therapy. It should be emphasized that seven of the 11 patients (results not shown) exhibited an increase in hemoglobin of more than $1 \mathrm{~g} / \mathrm{dl}$, demonstrating the presence of an iron deficiency. Thus, it appears that the inflammation-dependent component of the anemia is more intense among the patients with cholestasis than in the group with liver disease, but no cholestasis. Body iron content indicators should be interpreted taking account of the influence of the inflammation, which may result in an increase in ferritin unrelated to an increase in body iron reserves. Our results suggest that, in the management of anemia in patients with chronic liver disease, it is useful to perform a ferrous sulphate therapy test. It should be remembered that iron therapy for iron deficiency anemia should be continued for 2 months after normalization of hemoglobin in order to complete body iron deposits. ${ }^{14}$

Finally, it should be emphasized that patients with liver disease with cholestasis present more severe proteinenergy malnutrition than patients without cholestasis. The stature for age deficit is the most preeminent. It is necessary to widen knowledge on this subject, considering the need to improve the nutritional therapy afforded these patients since nutritional status is one of the principle prognostic factors. In turn, anemia is common among patients with chronic liver disease. Patients with disease without cholestasis present similar intestinal iron uptake to patients with iron deficiency anemia. Among patients with cholestasis, higher levels of ferritin and lower intestinal iron uptake suggest that, at least in part, anemia is related to inflammation, while the response to oral iron therapy indicates a coexisting iron deficiency.

\section{Acknowledgement}

We are grateful to Bristol-Myers Squibb for providing their Fer-in-sol product, which was used for oral iron therapy.

\section{References}

1. Kaufman SS, Murray ND, Wood RP, Shaw W Jr, Vanderhoff J. Nutritional support for the infant with extrahepatic biliary atresia. J Pediatr. 1987;110:679-86.

2. Klooster JK, Gregg DJ, Farrell MK, Heubi JE, Suchy FJ, Balistreri WF. Characterization of nutritional status in children with chronic liver disease. Pediatr Res. 1986;20:243-A.

3. Kaufman SS, Scrivner DJ, Guest JE. Preoperative evaluation, preparation, and timing of orthotopic liver transplantation in the child. Semin Liver Dis. 1989;9:176-83. 
4. Beath SV, Both W, Kelly DA. Nutritional support in liver disease. Arch Dis Child. 1993;69:545-9.

5. Deirdre AK. Nutritional factors affecting growth before and after liver transplantation. Pediatr Transp. 1997;1:80-4.

6. Chin SE, Shepherd RW, Thomas BJ, Gleghorn GJ, Patrick MK, Wilcox JA, et al. The nature of malnutrition in children with endstage liver disease awaiting orthotopic liver transplantation. Am J Clin Nutr. 1992;56:164-8.

7. Greer R, Lehnert M, Lewindon P, Cleghorn GJ, Shepherd RW. Body composition and components of energy expenditure in children with end-stage liver disease. J Pediatr Gastroenterol Nutr. 2003;36:358-63.

8. Moukarzel AA, Najim I, Vargas J, Mcdiarmid SV, Busuttil RW, Ament ME. Effect of nutritional status and outcome of orthotopic liver transplantation in pediatric patients. Transplant Proc. $1990 ; 22: 1560-3$.

9. Rodeck B, Melter M, Kardorff R, Hoyer PF, Ringe B, Burdelski M, et al. Liver transplantation in children with chronic and stage liver disease. Transplantation. 1996;62:1071-6

10. Ramaccioni V, Soriano HE, Arumugam R, Klish W. Nutritional aspects of chronic liver disease and liver transplantation in children. J Pediatr Gastroenterol Nutr. 2000;30:361-7.

11. Stephenson GR, Moretti EW, El-Moalem H, Clavien PA, TuttleNewhall JE. Malnutrition in liver transplant patients. Transplantation. 2001;72:666-70.

12. Cardoso AL, Porta G, Vieria MA, Carraza FR. Caracterização nutricional de crianças com colestase crônica. J Pediatr (Rio J). 1997;3:43-50.

13. Eckman JR. The liver and hematopoiesis in hepatology. In: Zakin D, Boyer TD, editors. Hepatology: a text book of liver disease. Philadelphia: W. B. Saunders; 1996. p. 685-890.

14. Morais MB, Suzuki HU, Machado NL, Fagundes Neto U. Avaliação de um teste simples de absorção intestinal de ferro na deficiência de ferro. J Pediatr (Rio J). 1992;68:48-53.

15. Morais MB, Suzuki HU, Corral JN, Machado NL, Fagundes-Neto U. Asymptomatic giardiasis does not affect iron absorption in children with iron deficiency anemia. J Am Col Nut. 1996;15: 434-8.

16. Scheuer PJ, Lefkowitch JH, editors. Cirrhosis. In: Liver biopsy interpretation. 5th ed. Philadelphia: W. B. Saunders; 1994. p. 135-51.

17. Maller ES. Jaundice. In: Altschler SM, Liacouras CA, editors. Clin Pediatr Gastroenterol. Oxfdord: Churchill Livingstone; 1998. p. 49-61.

18. Krause MV, Mahan LK, editores. Alimentos, nutrição e dietoterapia. 7a ed. São Paulo: Roca; 1985. p. 247-68.
19. Sabaté J. Estimación de la ingesta dietética: métodos y desafios. Med Clin. 1993;100:592-6.

20. Anção MS, Cuppary L, Ludisco E, Draibe AS, Sigulen D. Centro de Informática da UNIFESP - EPM. Sistema de apoio à nutrição, versão 2.5, 1995.

21. National Research Council (U.S.). Committee on dietary allowances. Recommended Dietary Allowances. 10th ed. Washington: National Academy of Sciences; 1989.

22. Jelliffe DB. Evaluación del estado de nutrición de la comunidad. Genebra: Organización Mundial de la Salud (Série de Monografias, 53); 1968.

23. Frisancho AR. Triceps skin fold and upper arm muscle size norms for assessment of nutritional status. Am J Clin Nutr. 1974;27:1052-8.

24. Dean AG, Dean JA, Coulombier D, Brendel KA, Smith DC, Burton A, et al. Epi Info, Version 6.0. Atlanta (GO): Centers of Disease Control and Prevention, Atlanta; 1994.

25. Frisancho A. Anthropometric standards for the assessment of growth and nutritional status. Ann Arbor: Univ. Michigan Press; 1993.

26. Sann L, Durand M, Picard J, Lasne Y, Bethenod M. Arm fat and muscle areas in infancy. Arch Dis Child. 1988;63:256-60.

27. Dallman PR, Siimes MA, Stekel A. Iron deficiency in infancy and childhood. Am J Clin Nutr. 1980;33:86-118.

28. Sokol RJ, Stall C. Anthropometric evaluation of children with chronic liver disease. Arn J Clin Nutr. 1990;52:203-8.

29. Holt RI, Broide E, Buchanan CR, Miell JP, Backer AJ, Mowat AP, et al. Orthotopic liver transplantation reverses the adverse nutritional changes of end-stage liver disease in children. Am J Clin Nutr. 1997;65:534-42.

30. Rodeck B. Improvement of growth after growth hormone treatment in children who undergo liver transplantation. J Pediatr Gastroenterol Nutr. 2000;31:286-90.

Correspondence:

Mauro Batista de Morais

Rua Pedro de Toledo, 441

CEP 04039-031 - São Paulo, SP, Brazil 